\title{
Subcutaneous Sarcoidosis of the Face
}

\author{
Dubravka Bosnić ${ }^{1}$, Marko Barešić ${ }^{1}$, Dinko Bagatin ${ }^{2}$ and Ivana Ilić ${ }^{3}$
}

\begin{abstract}
Subcutaneous sarcoidosis (previously referred to as Darier-Roussy sarcoidosis) is an unusual and atypical form of sarcoidosis. It is characterized by formation of the noncaseating granulomas in the subcutaneous tissue with no other systemic manifestations present. Sometimes subcutaneous granulomas are the first sign of systemic sarcoidosis. We report a case of a 51-year-old woman with an isolated form of subcutaneous sarcoidosis with no apparent sign of any other systemic manifestations. The final diagnosis should be made by excluding all other more common diseases. As spontaneous remissions have been reported, regular follow-ups for the first three to six months are advisable. If the remission does not take place within the given period of time or the symptoms progress rapidly, glucocorticoids are the drugs of choice. After improvement is seen, slow tapering of glucocorticoids is advisable.
\end{abstract}

Key words: subcutaneous sarcoidosis, subcutaneous nodules, face, facial edema

(Inter Med 49: 589-592, 2010)

(DOI: 10.2169/internalmedicine.49.2930)

\section{Introduction}

Sarcoidosis is a rare multisystemic disease of unknown origin that affects women more frequently than men. It is characterized by formation of noncaseating granulomas. The granulomatous inflammation can affect practically every organ in the body. The most frequent presenting manifestations involve the respiratory tract (hilar lymphadenopathy, interstitial lung disease and alveolitis, pulmonary infiltrates), eye (acute anterior uveitis), skin (plaques, annular lesions, erythema nodosum, lupus pernio, granulomas of the dermis and subcutaneous tissue, infiltration of the scars and tattoos), liver and spleen (granulomatous hepatitis, hepatosplenomegaly), heart (infiltrative cardiomyopathies, conduction disturbance, pericarditis, heart failure), nervous system (both central and peripheral) and musculoskeletal organs. Clinical, laboratory, radiological, pathohistological and nuclear medicine (gallium scan) findings are crucial for the diagnosis of sarcoidosis. Spontaneous resolution has been reported frequently, so patients should be monitored for the first 3 to 6 months. In well-established and progressive disease, glucocorticoid therapy is indicated (high doses with slow tapering). Several novel therapies are currently under research (1).

\section{Case Report}

A 51-year-old woman had a history of implantation of silicon in both lips for cosmetic purpose 20 years previously. Several months after implantation, the silicon implants were completely removed. She had had no serious illnesses or conditions requiring hospitalization. She did not use any medication, tobacco, alcohol or illicit drugs. The patient presented with a 5-month history of swelling of the lips, eyelids and cheeks, but never had any swelling of the tongue, difficulties in breathing or changes in the skin above the affected areas (Fig. 1). She was a lawyer who had frequent contacts with people and the current cosmetic facial defect made her unable to function in everyday life. A detailed outpatient and hospital checkup revealed only slightly accelerated ESR and a mild thrombocytosis. Other blood tests (complete blood count, liver and renal function tests), thyroid gland status, immunological findings (C1-esterase inhibitor levels, ANA, ENA, ANCA, complement levels), protein electrophoresis (no signs of monoclonal gammapa-

\footnotetext{
${ }^{1}$ Division of Clinical Immunology and Rheumatology, Department of Medicine, University Hospital Center Zagreb, Zagreb, Croatia, ${ }^{2}$ Department of Surgery, University Hospital Center Zagreb, Kispaticeva, Zagreb, Croatia and ${ }^{3}$ Department of Pathology and Cytology, University Hospital Center Zagreb, Zagreb, Croatia
}

Received for publication September 24, 2009; Accepted for publication December 2, 2009

Correspondence to Dr. Marko Barešić, markobaresic@gmail.com 


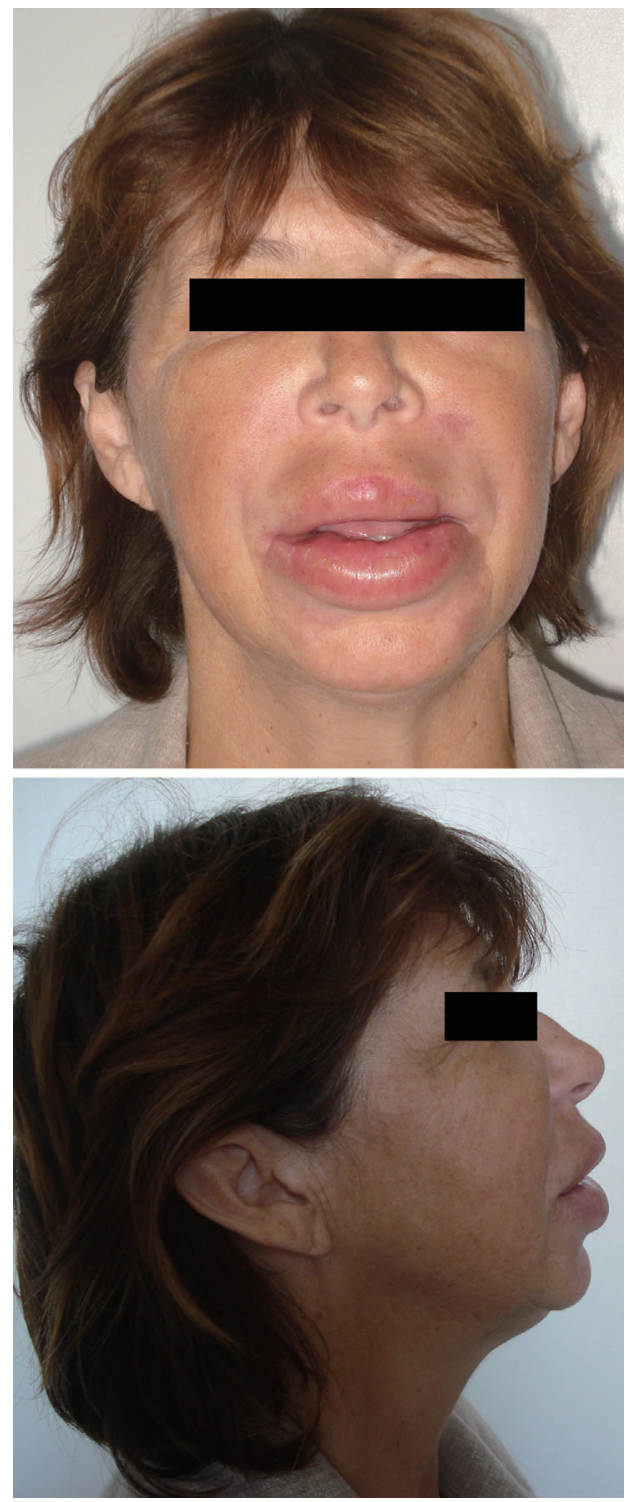

Figure 1. Patient before the treatment.

thy), allergy testing, stool sampling (for parasites and bacteria) were normal (Table 1). Ultrasonography of the lips showed edematous tissue and hypoechogenous zones (Fig. 2). MRI of the face was conclusive with edema and diffuse infiltrates of the soft tissue of the lips without signs of necrosis and foreign bodies (Fig. 3). No definitive diagnosis was reached and an allergic reaction was the most presumptive cause. A treatment attempt with intermittent low dose glucocorticoids $(10 \mathrm{mg}$ of per os prednisone, tapered after 5 day to $5 \mathrm{mg}$ and after 10 days discontinued) led to only a partial reduction in the facial edema with recurrence soon after the steroid was tapered. A surgical reduction of the lips was discussed. The patient was referred to our department for additional checkup. A detailed evaluation excluded the compression of the superior vena cava and the presence of hilar lymphadenopathy and any other pulmonary pathology (CT of the thorax and neck), visible heart abnormalities and amyloidosis. Elevated levels of ACE and hypercalciuria with normal serum calcium levels made the diagnosis of sarcoidosis very probable (Table 1). A further inves-
Table 1. Laboratory Findings

\begin{tabular}{|c|c|}
\hline Test & Result \\
\hline ESR $(\mathrm{mm} / \mathrm{h})$ & 30 \\
\hline CRP (mg/L) & Normal \\
\hline CBC* & Normal \\
\hline Platelets & 466 \\
\hline LFT** & Normal \\
\hline RFT*** & Normal \\
\hline Electrophoresis & Normal \\
\hline IgE & Normal \\
\hline Thyroid status & Normal \\
\hline ANA & Negative \\
\hline ENA & Negative \\
\hline ANCA & Negative \\
\hline $\begin{array}{c}\text { Complement } \\
\text { level }\end{array}$ & Normal \\
\hline $\begin{array}{l}\text { C1-esterase } \\
\text { inhibitor }\end{array}$ & Normal \\
\hline $\begin{array}{l}\text { Allergologic } \\
\text { testing }\end{array}$ & Negative \\
\hline $\begin{array}{l}\text { Stool sample } \\
\text { (bacteria, } \\
\text { fungi, } \\
\text { parasites) }\end{array}$ & Negative \\
\hline ACE (U/L) & 134 \\
\hline $\begin{array}{l}\text { Serum } \\
\text { Calcium }\end{array}$ & Normal \\
\hline $\begin{array}{c}\text { Urine Calcium } \\
\text { (dU) }\end{array}$ & 8.73 \\
\hline
\end{tabular}

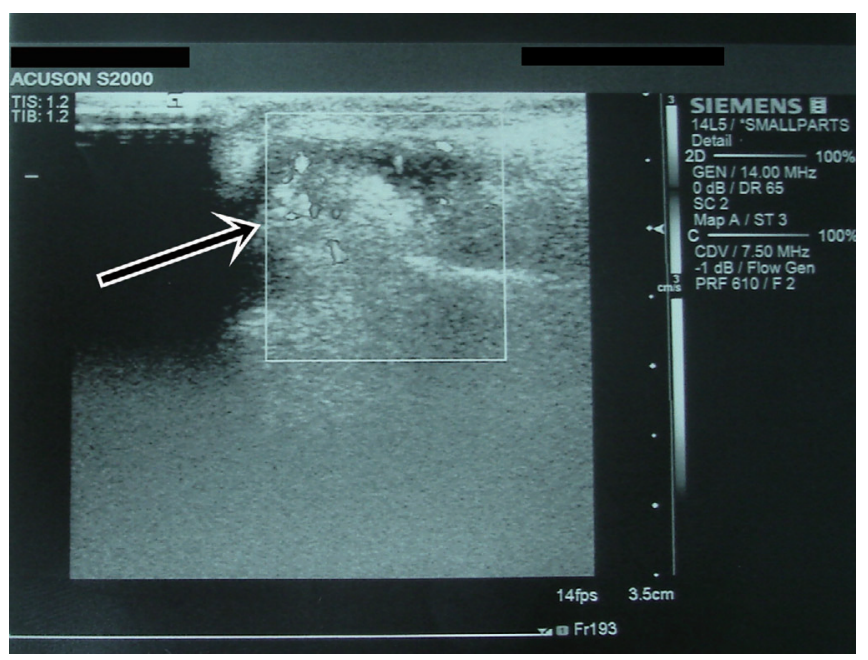

Figure 2. Ultrasonography of the lip. Arrow indicates the subcutaneous node in the lip.

tigation excluded pulmonary, heart and other systemic manifestations of sarcoidosis. The biopsy of a palpable subcutaneous tissue in the affected area around the lips and pathohistological findings revealed chronic inflammatory infiltrates of the dermis and subcutaneous fat with noncaseaeting epithelioid cell granulomas of different sizes with giant cells (Fig. 4). No necrosis was found in the tissue specimen and foreign bodies (such as residual silicon or exogenous silica) 


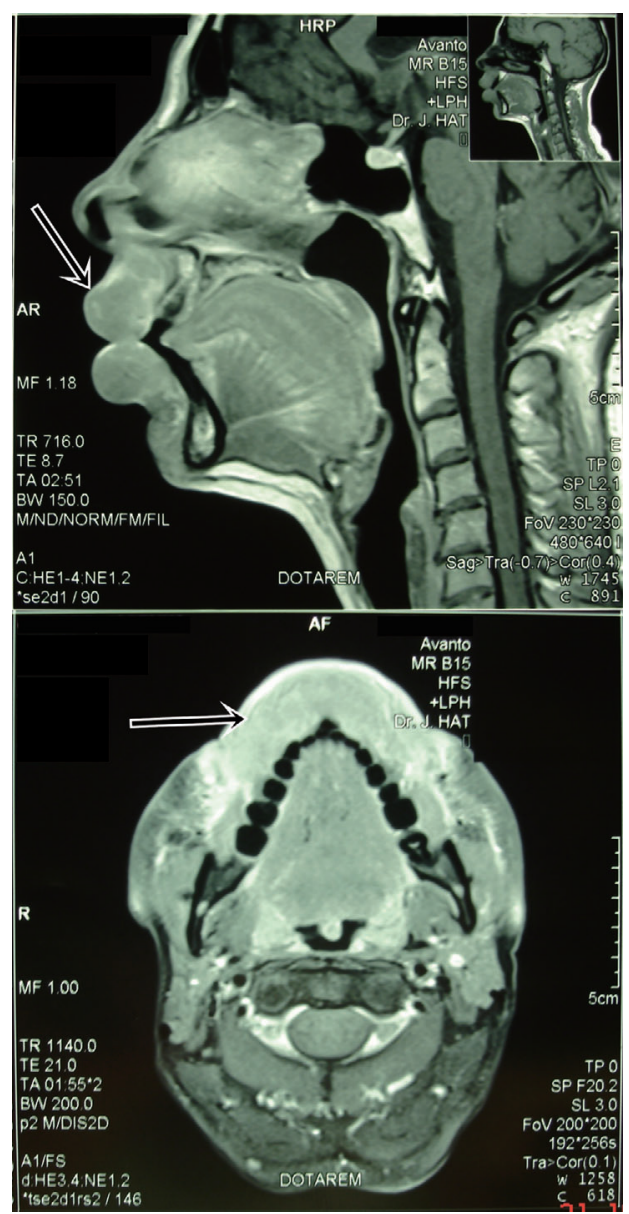

Figure 3. MRI of the face. Arrows indicate diffuse infiltrates of the soft tissue of the lips.

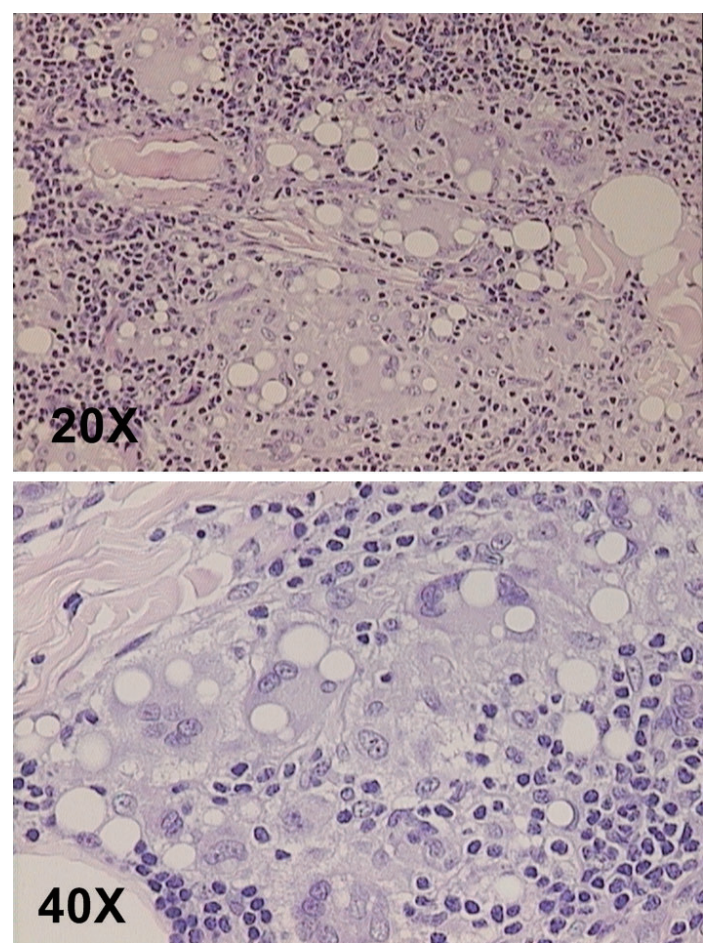

Figure 4. Hemalaun-eosin staining (magnification 20x and $40 \times$ ) shows infiltrates of the dermis and subcutaneous fat with granulomas.
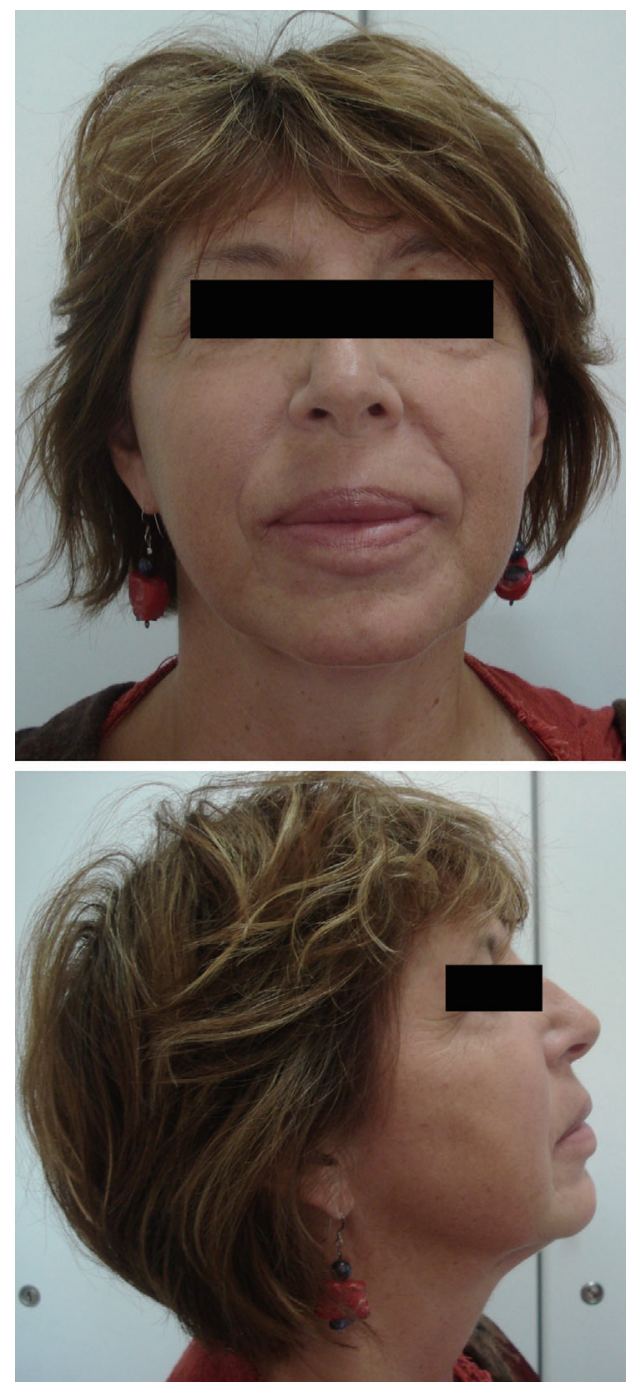

Figure 5. Patient after 3 weeks of treatment.

were excluded by polarized light microscopy. Special staining of the tissue, performed to rule out other causes of granulomas (mycobacteria and fungi), came back negative. The final diagnosis of subcutaneous sarcoidosis was reached. Taking into account the long period needed to reach the correct diagnosis and the fact that spontaneous resolution did not occur, high doses of glucocorticoids were initiated (methylprednisolone $125 \mathrm{mg}$ parenterally from day one to day three; methylprednisolone $60 \mathrm{mg}$ parenterally from day four to day six and later methylprednisolone 32 mg per os). Several days after the start of steroids, the edema of the lips and cheeks gradually started to diminish. In the follow-up period the facial edema subsided almost completely (Fig. 5) and the steroid levels were slowly tapered as well.

\section{Discussion}

Skin involvement is quite common in patients with systemic sarcoidosis (approximately 25-35\%); it presents as macules, papules and plaques on the neck, upper back, trunk and extremities, on scars and tatoos. These efflorescences are more cosmetic rather than life-threatening lesions (2). 
On the other hand, subcutaneous sarcoidosis (in the past referred as Darier-Roussy sarcoidosis) with no elements of systemic involvement is a rare condition. It has been reported in only 1.4 to $6 \%$ of patients with systemic sarcoidosis $(3,4)$. Several isolated cases of subcutaneous involvement have been described mostly with trunk and extremities as the predilection sites, but a thorough search of the current medical literature showed no cases like the present case with the face being the only affected area. Clinical characteristics include multiple, asymptomatic, palpable subcutaneous nodules. The skin above the nodules is unchanged. The diagnosis is confirmed by a pathohistological finding of noncaseating granulomas in the obtained tissue sample. Spontaneous remissions have been seen and sometimes the subcutaneous form is the first sign of the systemic presentation of the disease (5). The diagnosis should be reached only after excluding other more common diseases with similar clinical manifestations. A differential diagnosis of our patient's facial edema included angioedema, allergic reaction to implanted silicon or exogenous substances or medications, parasitic infestations, compression of the vascular structures in the neck or upper thorax, amyloidosis, hypothyroidism, hypoproteinemic edema, lysosomal storage diseases and other similar conditions. Another rare condition which should be taken into account is formation of noncaseating granulomas due to unintentional deposition of birefringent crystalline foreign material like silica. Silica granulomas can be detected by polarized light microscopy and energy-dispersive X-ray analysis $(6,7)$. Kim et al discussed the role of foreign bodies (like calcium, phosphorus, silicon, and aluminum) in the pathogenesis of granuloma formation in the skin of patients with systemic sarcoidosis. They concluded that the foreign bodies may be a provocative stimulus for granuloma formation in selected cases of sarcoidosis (8). The present patient had a silicone-based material implanted in her lips 20 years previously (which was removed several months later). We can not completely exclude the possibility of residual silicone being responsible for the formation of the subcutaneous granulomas, although it is quite unlikely. We would expect the granulomas to form sooner during the long period of 20 years. Evidence against this hypothesis include negative residual silicone (or any other foreign material in the acquired tissue) detected by polarized light microscopy together with clinical and laboratory data. After the correct diagnosis is reached, a detailed checkup of other organs is needed to confirm or rule out a systemic presentation. Glucocorticoids are the drugs of choice, adequately dosed and given during an adequate period of time. A slow tapering is advisable after the clinical and laboratory improvements are registered (9).

\section{Conflict of Interest}

There are no financial or other relationships that could lead to a conflict of interest regarding the manuscript.

\section{References}

1. West SG. Sarcoidosis. In: Rheumatology. Fourth edition. Volume 2. Hochberg MC, Silman AJ, Smolen JS, et al, Eds. Mosby Elsevier, Philadelphia, 2008: 1641-1651.

2. Iannuzzi MC, Rybicki BA, Teirstein AS. Sarcoidosis. N Engl J Med 357: 2153-2165, 2007.

3. Vainsencher D, Winkelmann RK. Subcutaneous sarcoidosis. Arch Dermatol 120: 1028-1031, 1984.

4. Marcoval J, Moreno A, Mana J, Peyri J. Subcutaneous sarcoidosis. Dermatol Clin 26: 553-556, 2008.

5. Marcoval J, Mana J, Moreno A, Peyri J. Subcutaneous sarcoidosis-clinicopathological study of 10 cases. Br J Dermatol 153: 790-794, 2005.
6. Mowry RG, Sams WM Jr, Caulfield JB. Cutaneous silica granuloma. A rare entity or rarely diagnosed? Report of two cases with review of the literature. Arch Dermatol 127: 692-694, 1991.

7. Lombardi T, Kuffer R, Dubrez B. Polishing-paste-induced silica granuloma of the gingiva. Dermatology 203: 177-179, 2001.

8. Kim YC, Triffet MK, Gibson LE. Foreign bodies in sarcoidosis. Am J Dermatopathol 22: 408-412, 2000.

9. Heller M, Soldano AC. Sarcoidosis with subcutaneous lesions. Dermatol Online J 14: 1, 2008.

(C) 2010 The Japanese Society of Internal Medicine http://www.naika.or.jp/imindex.html 